\title{
Building an Information Network System for Retirees in the Province Nineveh
}

Azza H. Zaki

College of Computer Science and Mathematics

University of Mosul

Received on: 08/10/2010

\section{Elham S. Mustafa \\ Computer Center}

Accepted on: 10/11/2010

\section{ABSTRACT}

Information systems has become an important tool for improving the working efficiency of the organizations that have tended to design and build information systems for the purpose of control for the vast amount of information necessary.

This research aims to provide information for retirees in the province of Nineveh, and to identify the requirements of official circles of the information. This system requires the use of distributed databases and a set of central computers linked to a computer by a communications network and more information can be exchanged between network elements.

Keywords: Information Network System, database.

$$
\begin{aligned}
& \text { بناء نظام شبكات معلومات لمتقاعدي محافظة نينوى }
\end{aligned}
$$

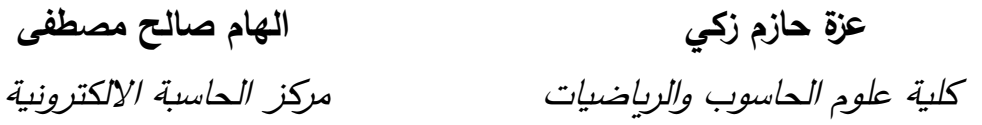

$$
\begin{aligned}
& \text { جامعة الموصل }
\end{aligned}
$$

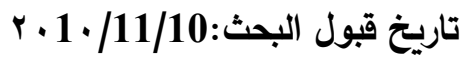

$$
\begin{aligned}
& \text { تاريخ استلام البحث:2010/10/8 }
\end{aligned}
$$

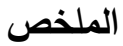

$$
\begin{aligned}
& \text { أضحت نظم المعلومات أداة هامـة من أدوات تحسين كفاءة العمل لذلك اتجهت المنظمات إلى تصميم } \\
& \text { وبناء أنظمة معلومات لغرض السيطرة على الكم الهائل من المعلومات الضرورية. } \\
& \text { يهدف هذا البحث إلى توفير معلومات عن المتقاعدين في محافظة نينوى وتحديد متطلبات الدوائر الرسمية من } \\
& \text { المعلومات .ويتطلب تحقيق هذا النظام استخدام قواعد بيانات الموزعة ومجموعة من الحواسيب مرتبطة بحاسوب } \\
& \text { مركزي بواسطة شبكة اتصالات وبشكل يمكن تبادل المعلومات بين عناصر الثبكة. } \\
& \text { الكلمات المفتاحية: نظام شبكات معلومات، قاعدة بيانات. } \\
& \text { الجانب النظري } \\
& \text { تقوم المؤسسات بتطوير وتثغيل العديد من نظم المعلومات الفرعية، مما يقود إلى تضخم حجم البيانات } \\
& \text { التي يجب أن تجمع وتخزن وتعالج، مما يعني ارتفاع تكلفة تخزين وتحديث والحفاظ على هذه البيانات، لذلك فئك }
\end{aligned}
$$

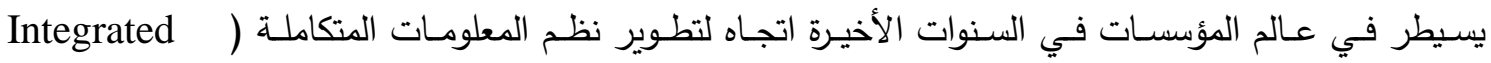

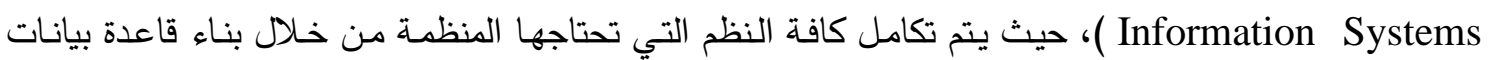


موحدة وعامـة، تتضـن كافـة البيانـات التي تحتاجها مختلف التطبيقات التي تفرضـها حاجـات إدارة المنظمـة من

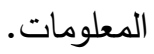

إن بناء قاعدة بيانات موحدة لكافة النظم ضمن المنظمة سوف يقلل من تكاليف القياس ويقلل أيضا من

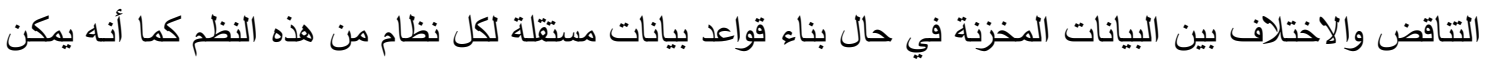

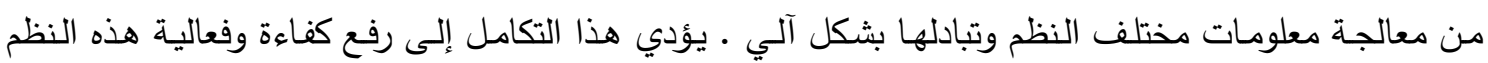
مجتمعة ويقلل من تكاليف عمليات التحديث المستمرة لمحتوى قاعدة البيانات. يتم تصميم قاعدة البيانات العامة والموحدة لدائرة تقاعد نينوى عبر النظر إلى الكيانات (Entities ) التي سوفي يتضدنها النظام، حيث يتم تضمين كل ملف من الملفات بجميع الحقول التي تهم كافة وظائف وأنشطة دائرة التقاعد

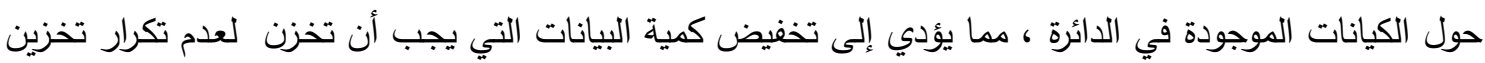
البيانات والمشاكل المرتبطة بها مثل مشاكل التحديث وخلو البيانات من التتاقض والاختلاف.

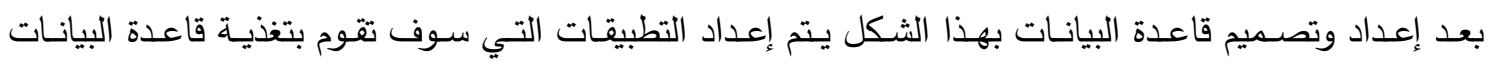

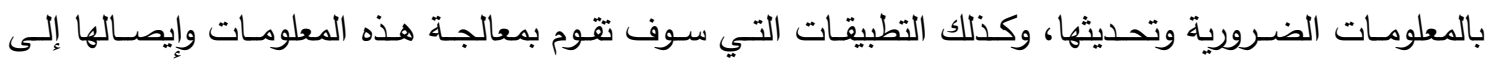

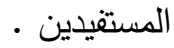

يتم إعداد هذه التطبيقات باستخدام أساليب المعالجة الآنية ( Real - Time- Processing ) في تحديث البيانات ومعالجتها ، حيث يتم إدخال البيانات مباشرة إلى الحاسوب فور وقوع الإجراء المعلوماتي عبر حوار بين

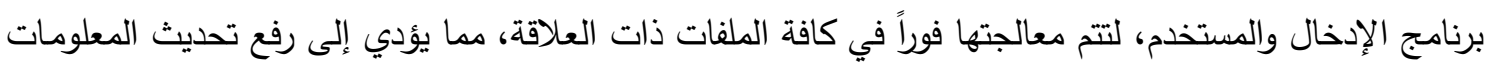
بثكل كبير يتطلب تحقيق مثل هذه الأنظمة استخدام قواعد البيانات الموزعة (Distributed Database ) ومجموعة من الحواسب مربوطة بحاسوب مركزي بواسطة شبكة اتصالات ( Network ) حيث تعمل كل الحواسب بنفس نظام إدارة قاعدة البيانات ( Oracle,2001) وبشكل يمكن في هذه الحالة تبادل المعلومات بين عناصر الشبكة

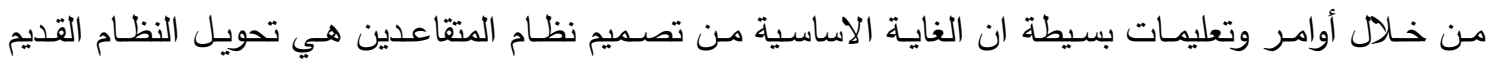
المستخدم من الصيغة التقليدية الى الصيغة البرمجية للمعلومات لتوفير سرعة ودقة وسرية في نقل البيانات ، النظام يقدم اسلوباً سهلاً مرناً في ادخال وتحديث وعرض ل...الخ للمعلومات وإدارة كل تلك العمليات تتم بواسطة مدير النظام (System Manager) مما سيختصر وقت الإنجاز من دون شك ويعطي إنتاجية عالية من خلال ما يلي : - النظام يوفر وسـائل سهلة وجيدة للتعامل مـع المعلومات الخاصـة عن المتقاعدين باستخدام برنامج الإدخال والبحث والتحديث لهعالجة المعلومات حيث تساعد في تسهيل عملية الإدخال والبحث وتتظيم والإحصائيات وإدارة

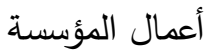
- - توفير الحماية للنظام للمحافظة على صحة المعلومات من أي تدخل غير مرغوب فيه .

$$
\text { - - متوفيره لتقارير وإحصاء ات مهمة جداً. }
$$

- إستخدام النظام لقاعدة البيانات Access لبناء الهيكل العام لما يوفر هذا النظام من تسهيلات برمجية.

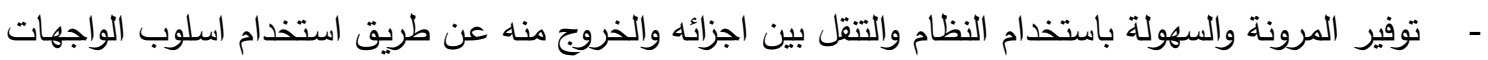
الصورية والنوافذ وتعريف عدد من مفاتيح وألاسهم تساهم في معالجة مشكلة عدم إلمام المستخدمين بالطريقة المثلى 
لاستخدام الحاسوب بالإضافة على قابلية النظام على تعديل بعض عناصر قاعدة المعلومات أو حذف عناصر أو إضافة عناصر جديدة بدون تأثير على مجمل معلومات القاعدة .

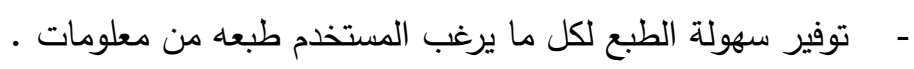

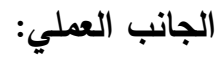 \\ الخطوة الاولى : تصميم نظام معلومات المتقاعدين}

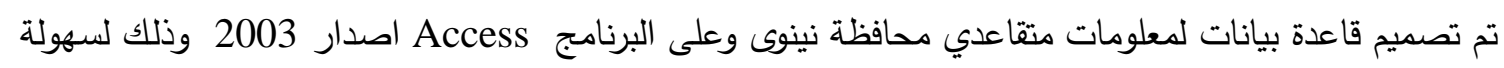
استخدامه من قبل موظفي دائرة تقاعد نينوى اذ ان العاملين على هذا النظام ليسوا من ذوي الاختصاص والتهات والخبرة في التعامل مع قواعد البيانات ـ واستخدمت عدة نماذج للنظام وهي كما ياتي:

النموذج الاول : النافذة الرئيسية للنظام ويحتوي هذا النموذج على اربعة اختيارات Command Button الاول

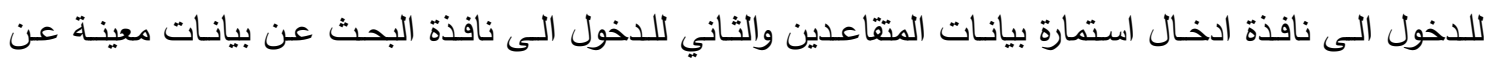
المنتسبين وحسب الحقل الذي يحدده المستخدم للنظام والاختيارالثالث للدخول الى نافذة التقارير والرابع اختيار

الخروج من النظام وكما موضح في الثكل (1).

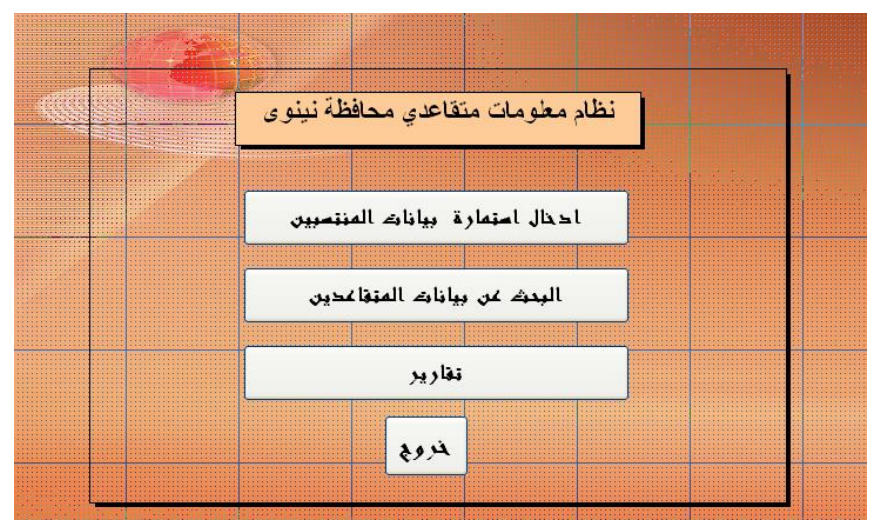

الثكل (1): النافذة الرئيسية لنظام معلومات المتقاعدين

النمـوذج الثاني : يمثل نافذة ادخال استمارة المتقاعدين والتي تحتوي على مجموعة من الحقول المهمة لغرض

الحصول على معلومات المتقاعد والاستفادة منها في تكوين التقارير وكما موضح في الثكل (2). 


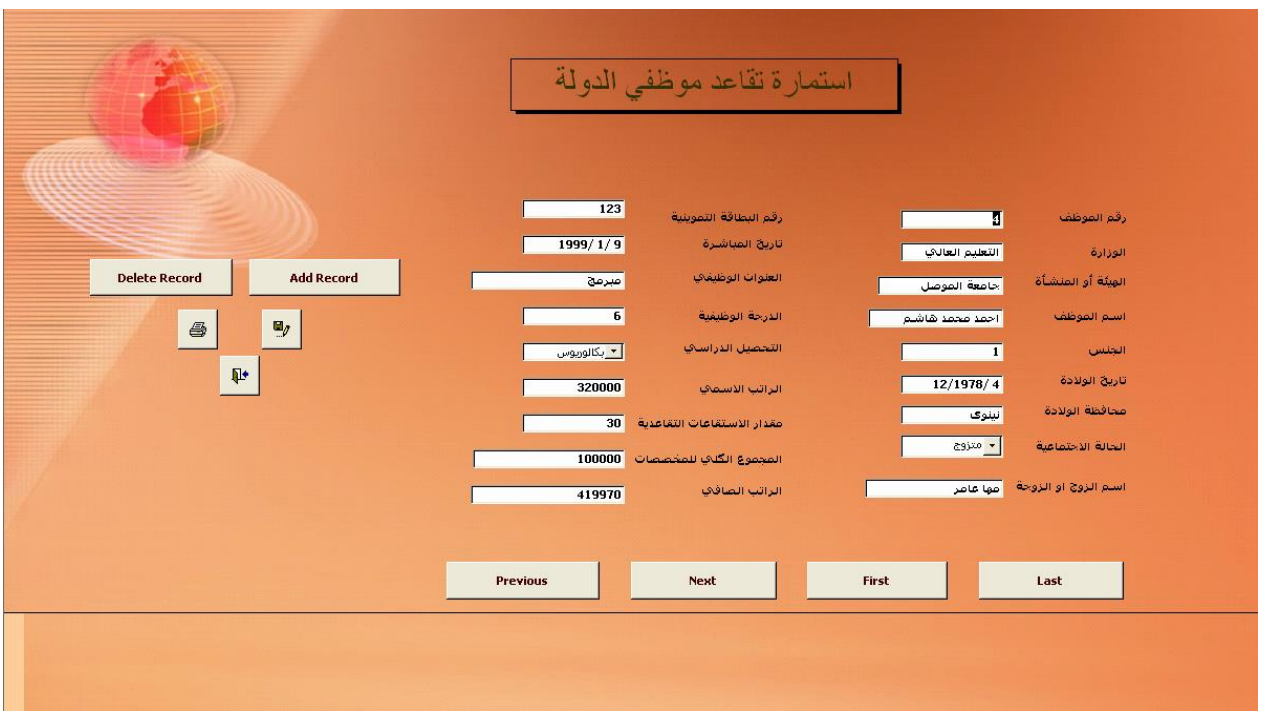

الثكل (2): نموذج الادخال لنظام معلومات المتقاعدين

النموذج الثالث : يمثل نافذة البحث عن بيانات المنتسبين وحسب الحقل الذي يحدده المستخدم للنظام (يمكن البحث

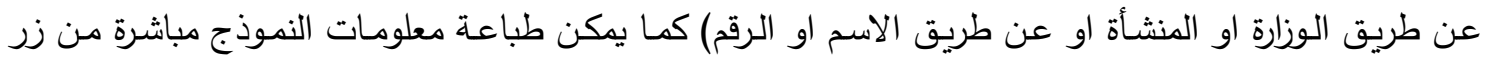

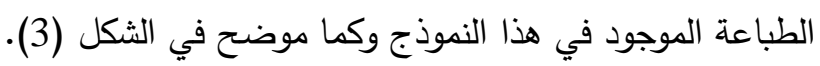

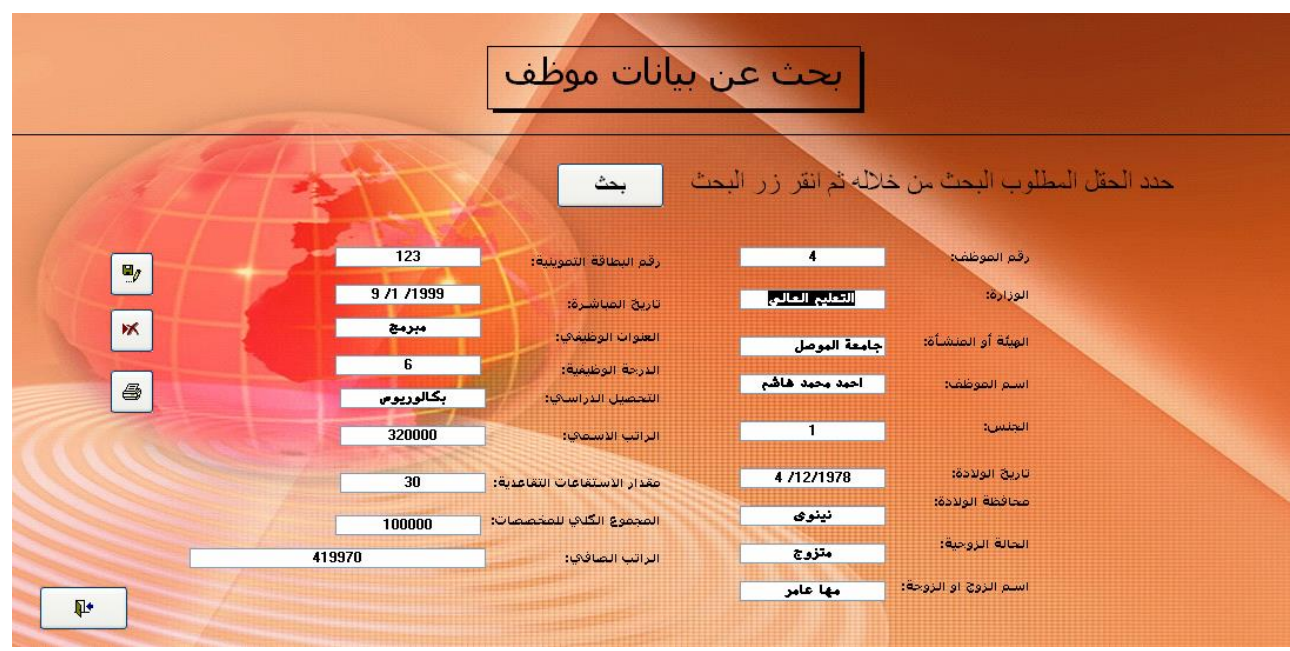

الثكل (3): نموذج البحث عن بيانات نظام معلومات المتقاعدين

النموذج اليرليع : يمثل نافذة التقارير وتحتوي هذه النافذة سبعة اختيارات Command Button الاول لعرض المنتسبين الذين تجاوزت رواتبهم 500000 (لقطع البطاقة التموينية) والثاني لعرض المنتسبين الذين تجاوزوا السن القانونية لغرض احالتهم للتقاعد والثالث لعرض المنتسبين حسب القدم وبقية التقارير تمثل منتسبي كل وزارة في تقرير خاص بها وبالامكان اضافة تقارير جديدة الى هذه النافذة وكذلك طباعة التقارير بصورة مباشرة وكما موضح

في الثكل (4). 


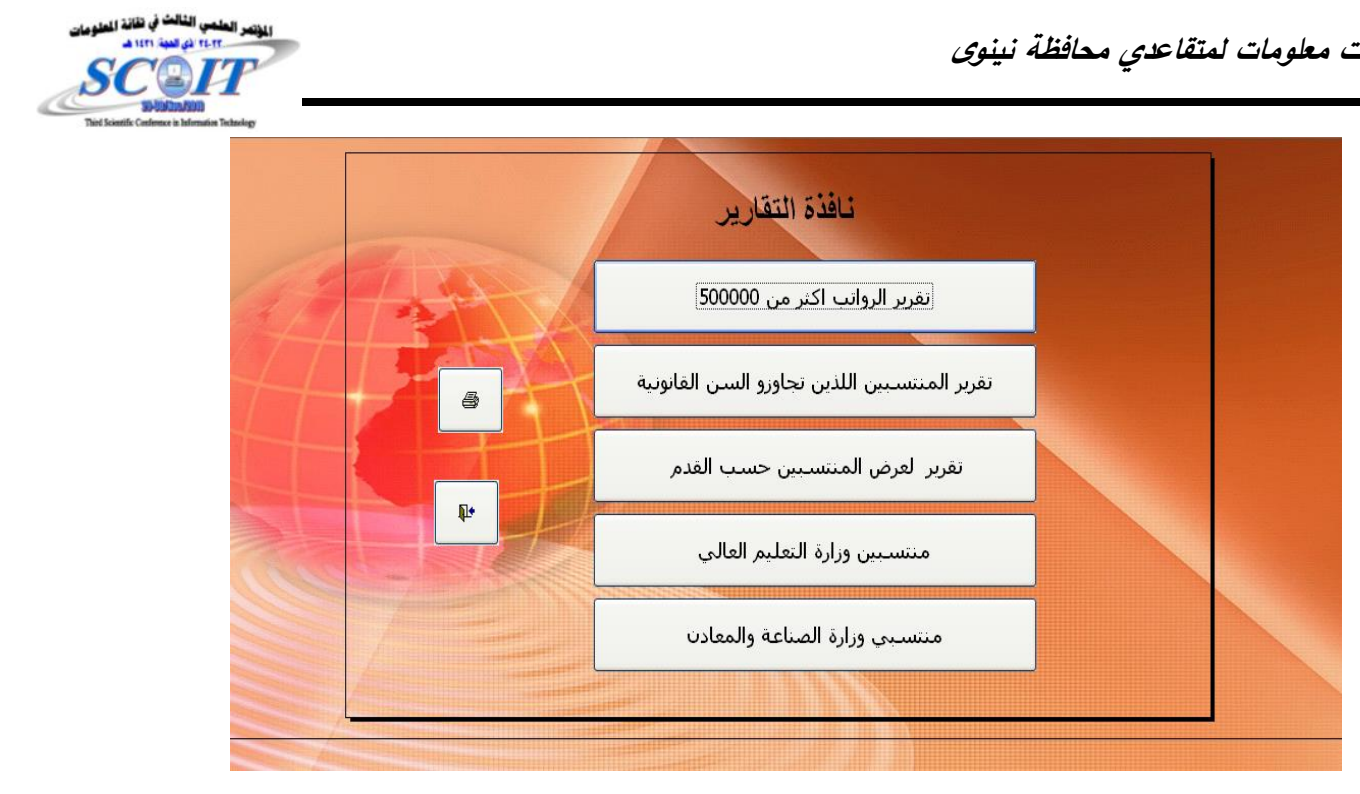

الشكل (4): الاحصائيات التي يمكن الحصول عليها من نظام معلومات المتقاعدين

ويوضتح الملحق (1) بعض الاحصائيات التي يمكن الحصول عليها من النظام •

SQL Server الخطوة الثانية: الاتصال بقواعد البيانات

Access الاتصال بقاعدة البيانات

سنوضـح فيمـا ياتي كيفيـة الاتصـال بقواعد البيانات SQ SQ Server في البدايـة لابد من ذكر طريقـة

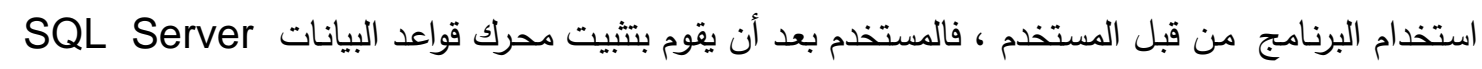

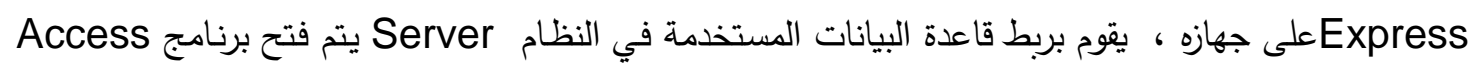
ومن ثم أنثاء قاعدة بيانات فارغة باسم Settings وتحفظ في مجلد باسم ( Databases ) في نفس مسار البرنامج.

الآن سنقوم بإنشاء جدول في قاعدة البيانات التي تم إنشاؤها باسم ( ConnectionToServer)

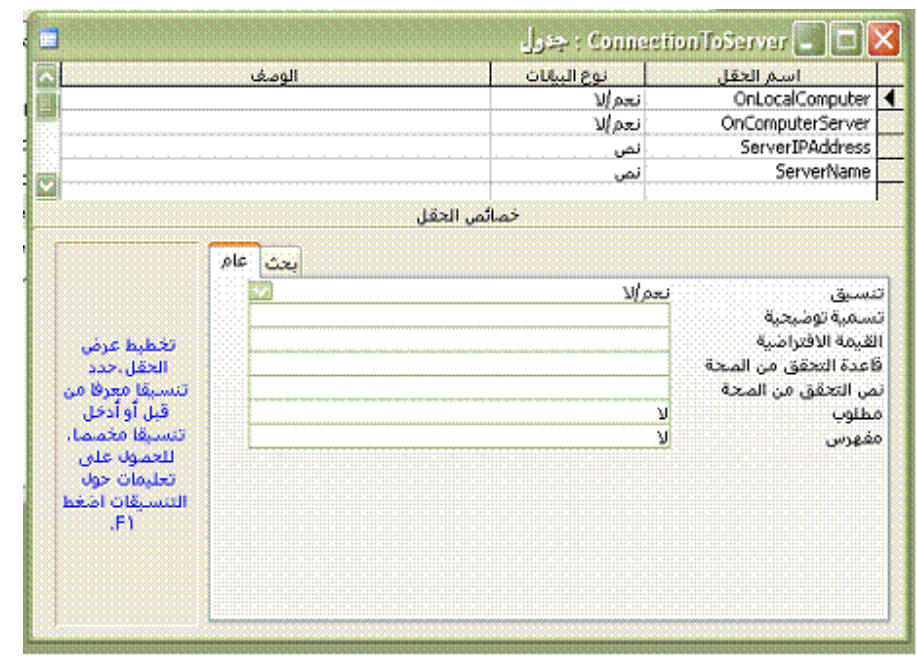

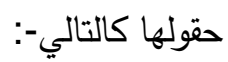

OnLocalComputer 
OnComputerServer

ServerIPAddress

ServerName

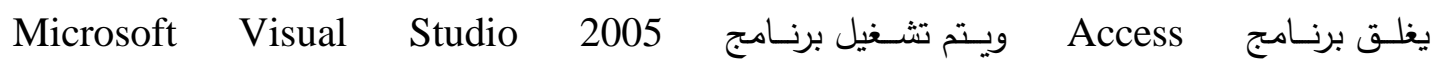

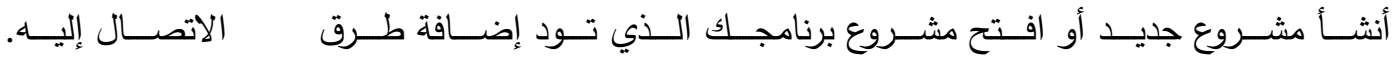

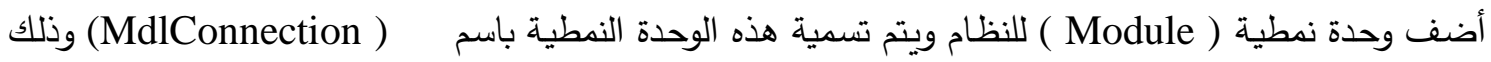
بفتح قائمة Project ثم و ... Add Module تحفظ الوحدة النمطية بجوار ملفات النظام.

في قسم التصريحات العامة في الـ Module يكتب الاتي:

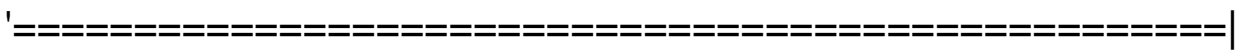

| ل هذا الموديل يقوم بعمل اتصال عام لقواعد البيانات'

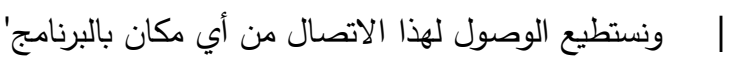

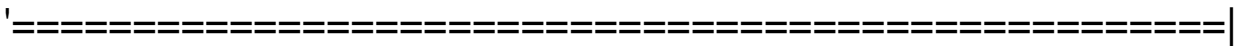

Option Explicit On

Imports System.Data

Imports System.Data.SqIClient

Imports System.Data.OleDb

Imports Microsoft.VisualBasic

Imports System.IO

وفي قسم التصريحات في الـ Module يكتب الرمز الاتي:

المتغيرات العامة للاتصال بخادم قواعد البيانات'

Public GSQLConnection As New SqIConnection

Public GSQLCmd As SqICommand = GSQLConnection.CreateCommand

Public GDA As SqIDataAdapter = Nothing

Public GDR As SqIDataReader = Nothing

Public GDS As New DataSet

Public GDT As DataTable

المتغيرات المحلية والتي ستحمل نوع الاتصال بخادم قواعد البيانات'

وذلك بعد معرفة نوع الخادم وبياناته من قاعدة البيانات المحلية '

Dim LocalComputer As Boolean = False

Dim ServerBylPAddress As Boolean = False

Dim ServerByName As Boolean = False

'== = = = = = = = = = = = = = = = = = = = = 
متغيران عامان يحملان قيمة عنوان الاي بي واسم السيرفر'

Public ServerIPAddress As String

Public ServerName As String

المتغيرات الخاصة بالاتصال بقواعد بيانات أكسس أي المحلية'

متغير الاتصال الأساسي' السئ'

Dim WithEvents Cn As New OleDbConnection

متغير نص الاتصال بقاعدة البيانات'

Dim CnStr As String

'متغير كائن الأوامر'

Dim WithEvents Cmd As New OleDbCommand

متغير نص جملة الاستعلام'

Dim Sql As String

'متغير يحمل مجموعة السجلات'

Dim DR As OleDbDataReader

بعدها نقوم بإنشاء إجراء عام كما في الرمز الاتي-:

Public Sub GeneralConnection()

إجلب نوعية الاتصال من قاعدة البيانات المحلية'

Call GetConnection()

فإذا كانت نوعيته من الخادم على الحاسوب المحلي'

If LocalComputer = True Then

استدعي إجراء الاتصال بخادم قواعد البيانات على الحاسوب المحلي'

Call ConnectToLocalServerComputer()

End If

If ServerByIPAddress = True Then

استدعي إجراء الاتصال بخادم قواعد البيانات عن طريق عنوان الأي بي'

Call ConnectTolPAddressServerComputer()

End If

If ServerByName = True Then

استدعي إجراء الاتصال بخادم قواعد البيانات عن طريق اسم السيرفر'

Call ConnectToNameServerComputer()

End If

End Sub

والآن سنقوم بكتابة الإجراء الذي يحتوي على الاتصال بقواعد بيانات Access لمعرفة خيارات المستخدم

Public Sub GetConnection() 


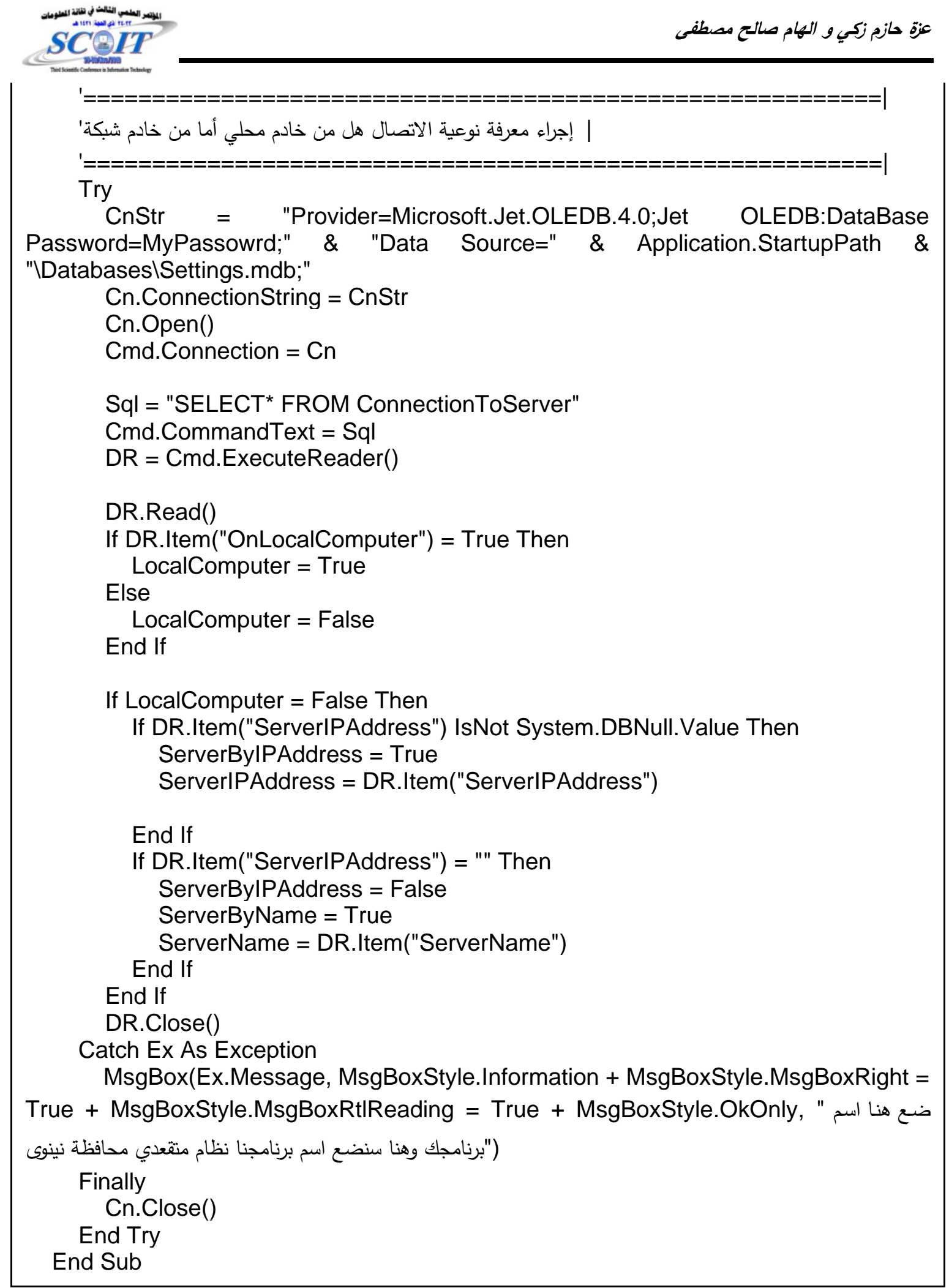

تكوين الإجراء الخاص بالاتصال بقاعدة بيانات SQ Server من خلال الحاسوب المحلي ( أي نفس الحاسوب الذي يعمل عليه النظام).

Public Sub ConnectToLocalServerComputer()

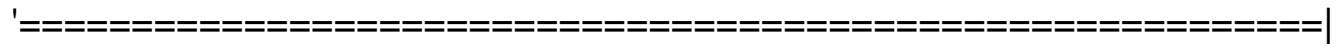

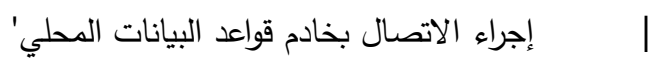




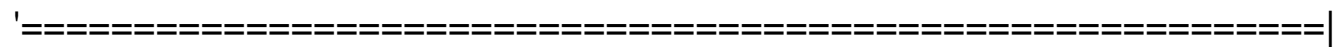

اجعل نص الاتصال هو الاتصال بالخادم المحلي'

Try

GSQLConnection.ConnectionString = "Data Source=" + My.Computer.Name + "ISQLExpress;Initial Catalog=نظام تقاعد موظفي الدولة:Integrated Security=SSPI;" GSQLConnection.Open()

Catch Ex As Exception

MsgBox(Ex.Message,

MsgBoxStyle.Information

MsgBoxStyle.MsgBoxRight $=$ True + MsgBoxStyle.MsgBoxRtIReading $=$ True + MsgBoxStyle.OkOnly, " نظام تقاعد موظفي الدولة ")

End Try

End Sub

هذا الإجراء بالاتصال بقاعدة بيانات SQL Server مستخدماً مجال الأسماء MY ومن ثم اسم الحاسوب كما في المقطع التالي. ( My.Computer.Name) في نص الاتصال تجد المقطع التالي نظام تقاعد موظفي الدولة =Catalog وهذا هو اسم قاعدة البيانات المطلوب الاتصال بها ثم قمنا بفتح الاتصال بقاعدة البيانات .

IP Address الاتصال بقاعدة البيانات من خلا

سنكتب الإجراء الذي يتصل بقاعدة البيانات من خلال الـIP Address

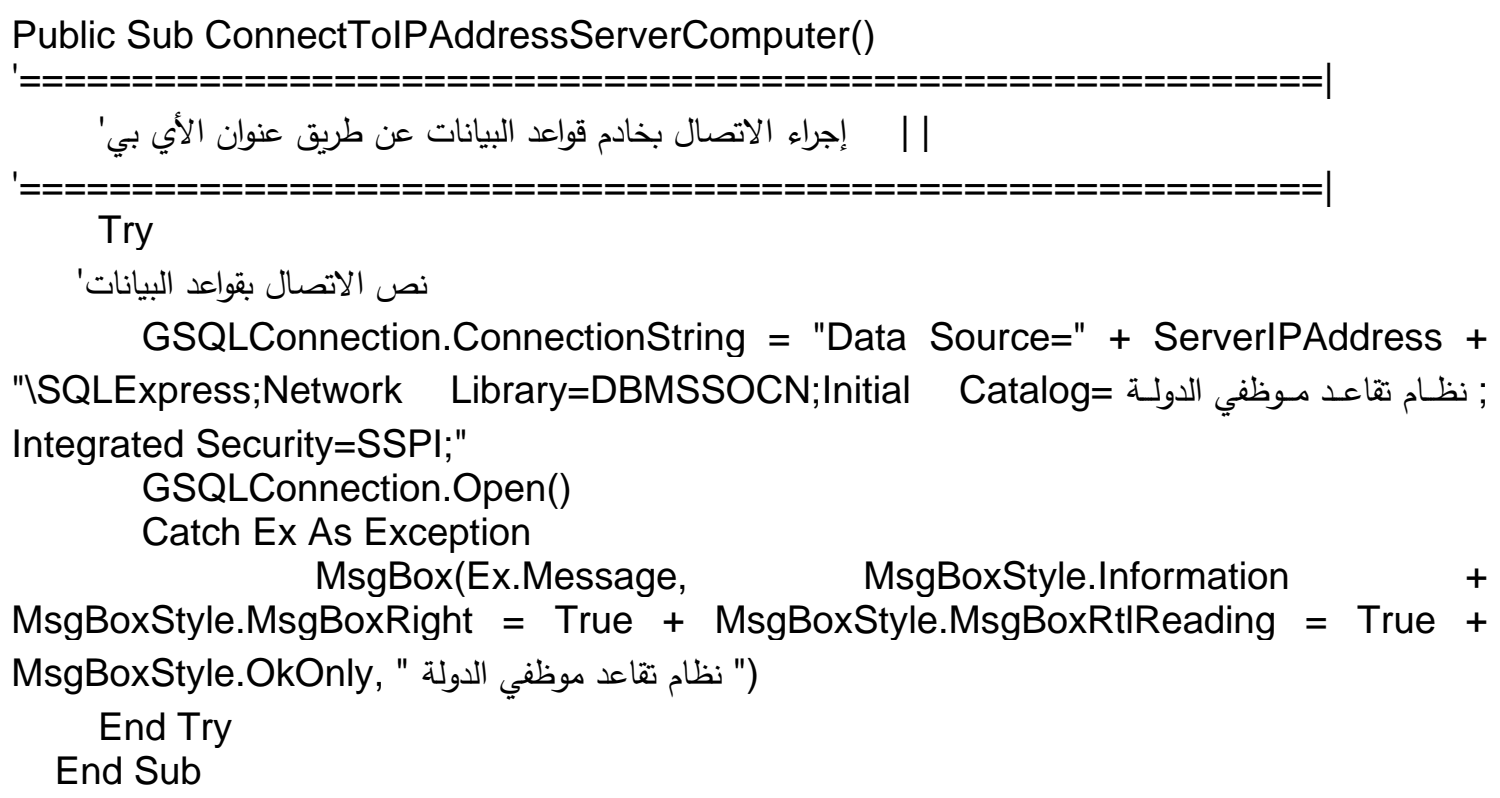

Access هنا استخدمنا في نص الاتصال المتغير ServerIPAddress والذي يحمل قيمته من قاعدة بيانات وهو خيار المستخدم. ثم قمنا بفتح الاتصال بقاعدة البيانات.

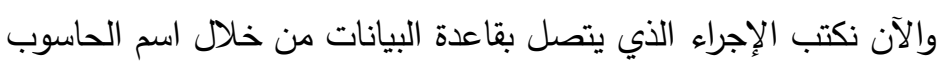

Public Sub ConnectToNameServerComputer()

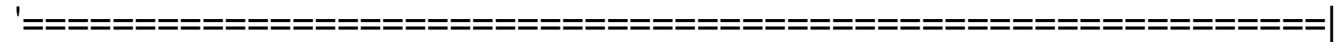

$$
\text { | | | | إجراء الاتصال بخادم قواعد البيانات عن طريق اسم السيرفر' }
$$




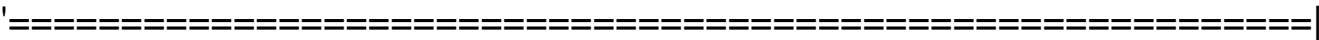

Try

نص الاتصال بقواعد البيانات'

GSQLConnection.ConnectionString = "Data Source=" + ServerName + "ISQLExpress;Initial Catalog= نظام تقاعد موظفي الدولة Integrated Security=SSPI;" GSQLConnection.Open()

Catch Ex As Exception

MsgBox(Ex.Message, MsgBoxStyle.Information + MsgBoxStyle.MsgBoxRight =

True + MsgBoxStyle.MsgBoxRtlReading=True+MsgBoxStyle.OkOnly," هنا اكتب اسم (برنامجك")

End If

End Try

End Sub

هنا استخدمنا في نص الاتصال المتغير ServerName والذي يحمل قيمته من قاعدة بيانات Access وهو خيار المستخدم ثم قمنا بفتح الاتصال بقاعدة البيانات. إلى هنا نكون قد قمنا بكتابة كل الإجراءات الخاصة

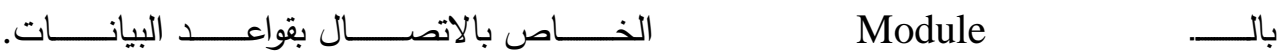
والآن نبدأ بتصميم النافذة التي ستتلقى خيارات المستخدم ومن ثم حفظها في قاعدة البيانات Access ويمكنك أن تجعها تظهر في أول مرة تثغيل لبرنامجك. صمم النافذة كما بالثكل التالي مستعيناً بالمعلومات التالية-:

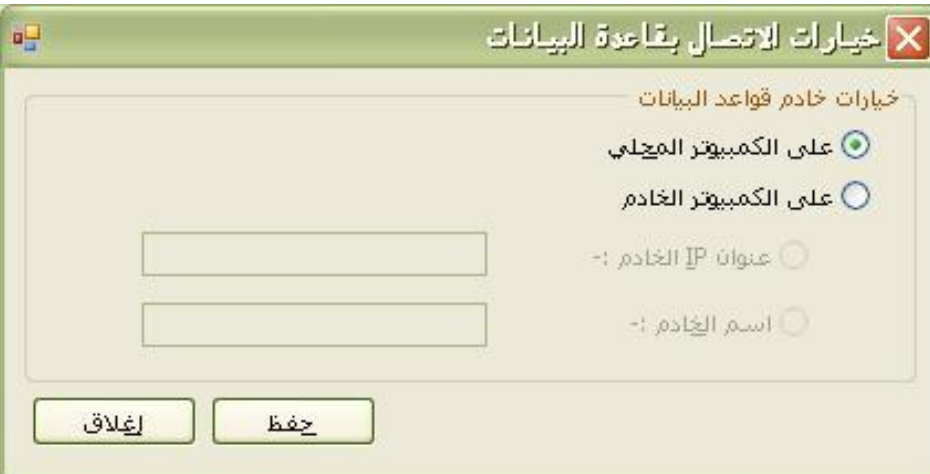

1- ضع على النافذة صندوق مجموعة Group Box وغير قيمة الـ Text إلى (خيارات خادم قواعد البيانات).

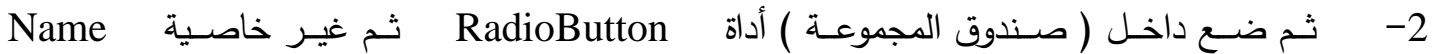
إلى RdoLocalComputer وغير قيمة الـ Text إل على الحاسوب المحلي.

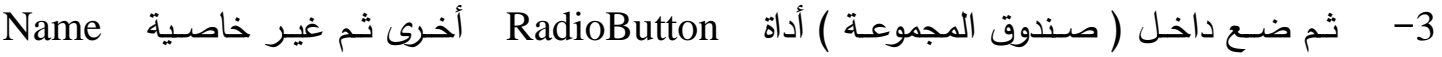
إلى RdoServerComputer وغير قيمة الـ Text إلى ( على الحاسوب الخادم).

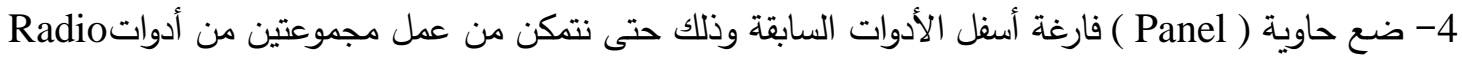

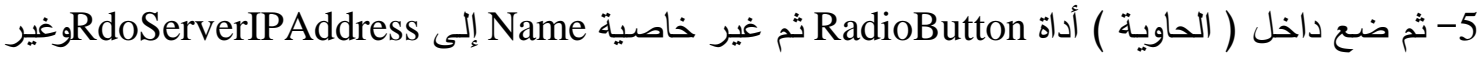
قيمة الـ Text إلى (عنوان IP الخادم ):- 
6- ثم ضـع داخل ( الحاوية ) أداة RadioButton أخرى ثم غير خاصية Name إلي RdoServerName وغير قيمة الـ Text إلى (اسم الخادم ):-

7- ضع بجوار الـ RadioButton الأولى في الحاوية أداة ( مربع نص Text Box ( ثم غير خاصية Name Enabled = False ثم إجلى TxtServerIPAddess

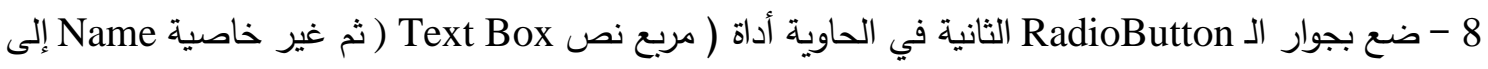

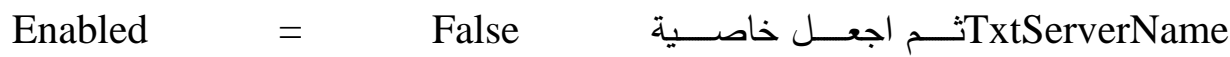
9- ضع (زري أوامر Command Button ( على النافذة وغير قيمة الـ Text للزر الأول إلى ( حفظ ) والثاني إلى ( إغلاق ).

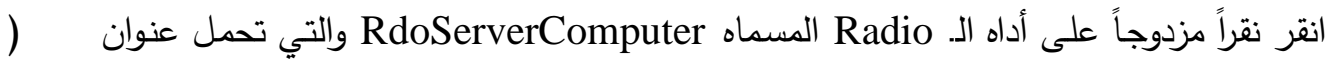

على الحاسوب الخادم )، وذلك لفتح نافذة الرمز على خاصية RdoServerComputer_CheckedChanged

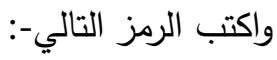
رمز:

If RdoServerComputer. . Checked $=$ True Then

RdoServerlPAddress. Enabled $=$ True

Me. TxtServerIPAddess. Enabled $=$ True

Me. RdoServerlPAddress. Checked $=$ True

Me.RdoServerName. Enabled $=$ True

Else

Me. RdoServerIPAddress. Enabled = False

Me. TxtServerIPAddess. Enabled $=$ False

Me. RdoServerName. Enabled = False

Me. TxtServerName. Enabled $=$ False

End If

في هذا الرمز نختبر اختيار المستخدم هل قام باختيار الخيار الثاني أي (على الحاسوب الخادم ) فإذا

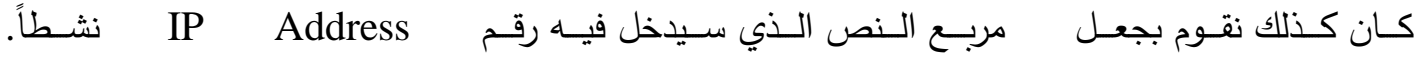
أما إذا رجع المستخدم عن اختياره واختار أداة الاختيار الأولى نجعل مربع النص (على الحاسوب الخادم )غير

الآن انقر نقراً مزدوجاً على أداه الـ Radio المسماه RdoServerName والتي تحمل عنوان ( اسم

الخادم )، وذلك لفتح نافذة الرمز على خاصيةRdoServerName_CheckedChanged واكتب الرمز التالي-:

If RdoServerName.Checked $=$ True Then

Me. TxtServerName. Enabled $=$ True

Me. RdoServerIPAddress. Checked = False

Me. TxtServerIPAddess . Enabled $=$ False 


\section{Else}

Me.TxtServerIPAddess.Clear()

Me. TxtServerName. Enabled $=$ False

Me. TxtServerIPAddess. Enabled $=$ True

End If

نقوم في هذا الرمز بمعرفة اختيار المستخدم فإذا اختار الخيار الثاني في الحاوية أي اختار (اسم الخادم

:- ) حينها نقوم بإلغاء تتشيط مربع نص ( رقم ( IP Address وجعله غير نشط ومن ثم تتشيط مربع نص ( اسم

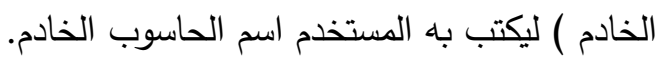

في قسم التصريحات العامة في النافذة نكتب الرمز التالي:

Option Explicit On

Imports System.Data

Imports System.Data.SqIClient

Imports System.Data.OleDb

Imports Microsoft.VisualBasic

Imports System.IO

Imports Microsoft.Win32

ثم اكتب في قسم التصريحات العامة للنافذة وتحت Public Class Form1 الرمز التالي-:

متغير الاتصال الأساسي' - (ماسي

Dim WithEvents Cn As New OleDbConnection

متغير نص الاتصال بقاعدة البيانات'

Dim CnStr As String

'متغير كائن الأوامر'

Dim WithEvents Cmd As New OleDbCommand

متغير نص جملة الاستعلام'

Dim Sql As String

'متغير يحمل مجموعة السجلات'

Dim DR As OleDbDataReader

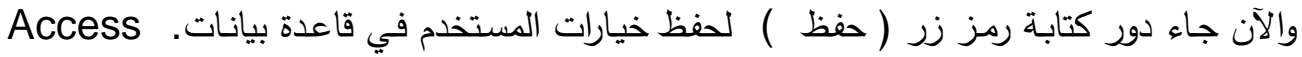

انقر نقراً مزدوجاً على زر ( حفظ) لفتح نافذة الرمز على الخاصية Button1_Click ثم اكتب الرمز التالي :

Try

CnStr = "Provider=Microsoft.Jet.OLEDB.4.0;Jet OLEDB:DataBase Password=MyPassowrd;" \& "Data Source=" \& Application.StartupPath \& "'Databases।Settings.mdb;"

Cn.ConnectionString $=$ CnStr

Cn.Open()

Cmd.Connection $=\mathrm{Cn}$ 
If Me.RdoLocalComputer. . Checked = True Then

Sql = "UPDATE ConnectionToServer SET OnLocalComputer=" \& True \& ",OnComputerServer=" \& False \& ",ServerIPAddress='" \& Me.TxtServerIPAddess.Text \& "'" \& ",ServerName="' \& Me.TxtServerName.Text \& "'"

End If

If Me.RdoServerComputer. .Checked $=$ True Then

If Me. RdoServerlPAddress. Checked = True Then

$\mathrm{Sql}=$ "UPDATE ConnectionToServer SET OnLocalComputer=" \& False \&

",OnComputerServer=" \& True \& ",ServerIPAddress="" \& Me.TxtServerIPAddess.Text \& "'" \& ",ServerName="' \& Me.TxtServerName.Text \& "'"

End If

If Me.RdoServerName. Checked $=$ True Then

Sql = "UPDATE ConnectionToServer SET OnLocalComputer=" \& False \& ",OnComputerServer=" \& True \& ",ServerIPAddress='" \& Me.TxtServerIPAddess.Text \& "'" \& ",ServerName="' \& Me.TxtServerName.Text \& "'"

End If

End If

Cmd.CommandText $=\mathrm{Sql}$

Cmd.ExecuteNonQuery()

MsgBox("تم حفظ خيـارات المستخدم في قاعدة البيانـات", MsgBoxStyle.Information + MsgBoxStyle.MsgBoxRight = True + MsgBoxStyle.MsgBoxRtIReading = True + MsgBoxStyle.OkOnly, " نظام تقاعد موظفي الدولة")

Me.Button1.Enabled $=$ False

Catch Ex As Exception

MsgBox(Ex.Message)

Finally

Cn.Close()

End Try

$$
\begin{aligned}
& \text { في هذا الرمز نقوم بحفظ خيارات المستخدم لاختياره لنوع خادم قواعد البيانات وذلك في قاعدة البيانات }
\end{aligned}
$$

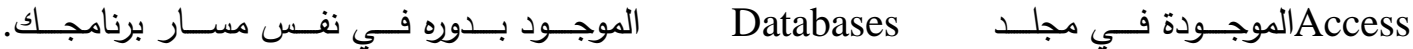

اكتب الرمز التالي في حدث النقر على زر أمر ( إغلاق ) لإغلاق نافذة خيارات خادم قواعد البيانات ومن ثم فتح

SQL Server :- نافذة الاتصال بقاعدة البيانات

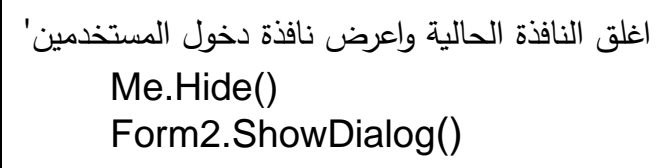

يجب في هذه المرحلة أن تقوم بعمل قاعدة البيانات SQ ومن ثم ربطها بخادم SQL Server Server Express، كما يجب أن تحتوي قاعدة البيانات على جدول باسم Users / بها حقلين الحقل الأول سمه فئ 
الآن صمم النموذج الثاني كما بالثكل التالي-:

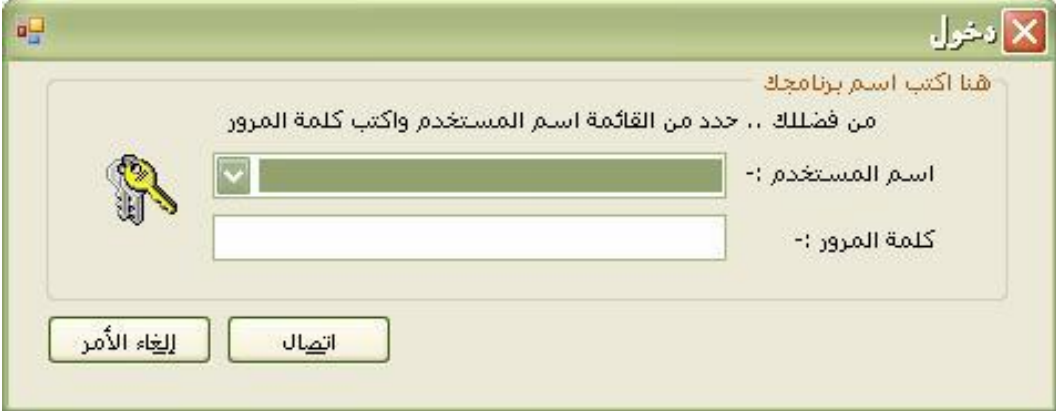

بحيث تجعل اسم القائمة التي سيكون فيها أسماء المستخدمين ( CmbUserName) وتجعل اسم مربع النص الذي يتلقى كلمات المرور باسم ( TxtPassword) ، ولا تتسى أن تجعل قيمة الخاصية Password Char لمربع

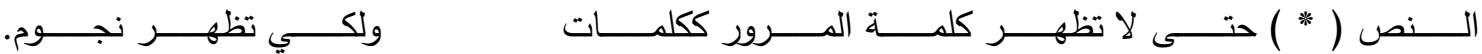

اجعل اسم الأمر ( اتصال ) باسم ( CmdConnect) والأمر ( إلغاء الأمر ) باسم. ( CmdCancel ) والآن سنكتب رمز الأمر (اتصال ) ، انقر نقراً مزدوجاً على أمر اتصال لفتح نافذة الرمز كما بالثكل التالي

CmdConnect Enabled $=$ False

Cursor $=$ Cursors. WaitCursor

التأكد من أن المستخدم قد اختار اسم مستخدم من القائمة'

If Me.CmbUserName. Text = "' Then

MsgBox(" من فضلك .. اختر اسم الستخدم من القائمسة " MsgBoxStyle.Information + MsgBoxStyle.OkOnly + $\quad$ MsgBoxStyle.MsgBoxRtlReading + MsgBoxStyle.MsgBoxRight, "كتب اسم برنامجك")

CmdConnect. Enabled $=$ True

Cursor $=$ Cursors. Default

Exit Sub

End If

If Me.TxtPassword. Text = "" Then

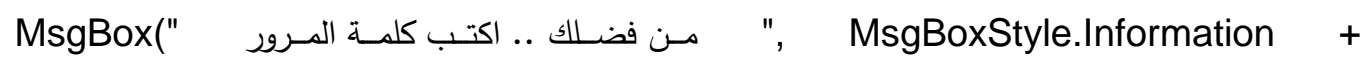
MsgBoxStyle.OkOnly + $\quad$ MsgBoxStyle.MsgBoxRtIReading + MsgBoxStyle.MsgBoxRight, "اكتب اسم برنامجك")

CmdConnect. Enabled $=$ True

Me.TxtPassword.Focus()

Cursor $=$ Cursors. Default

Exit Sub

End If

استدعاء الاتصال العام بقواعد بيانات السيرفر'

Try

معرفة حالة الاتصال العام فإذا كان غير مغتوح'

If GSQLConnection.State <> ConnectionState.Open Then

عندها استدعي إجراء الاتصال العام من مديول الاتصال' 
GeneralConnection()

End If

If GSQLConnection.State = ConnectionState.Open Then

GDR = Nothing

GSQLCmd.CommandType = CommandType. Text

GSQLCmd.CommandText = "SELECT* FROM Users WHERE UserID LIKE

"' \& Me.CmbUserName.Text \& "\%'"

GDR $=$ GSQLCmd.ExecuteReader

GDR.Read()

في حالة مساواة كلمة المرور المدخلة لكلمة المرور الموجودة في قاعدة البيانات'

If GDR.Item("Password") = Me.TxtPassword.Text Then

MsgBox(" انت الآن متصل بقاعدة البيانات بنجاح " MsgBoxStyle.Information +

MsgBoxStyle.OkOnly + + MsgBoxStyle.MsgBoxRtlReading +

MsgBoxStyle.MsgBoxRight, "اكتب اسم برنامجك")

End If

إغلق كائن قارئ البيانات'

GDR.Close()

Elself GDR.Item("Password") <> Me.TxtPassword.Text Then GSQLConnection.Close()

MsgBox(" من فضلك .. اكتب كلمة المرور الصحيحة " MsgBoxStyle.Information +

MsgBoxStyle.OkOnly + $\quad$ + MsgBoxStyle.MsgBoxRtIReading +

MsgBoxStyle.MsgBoxRight, "اكتب اسم برنامجك")

Me.TxtPassword.Focus()

End If

GDR.Close()

Catch ex As Exception

GSQLConnection.Close()

Finally

CmdConnect. Enabled $=$ True

Cursor $=$ Cursors. Default

End Try

لنكتب رمز إجراء جلب اسماء المستخدمين من قاعدة البيانات ووضعهم في قائمـة المستخدمين كما يلي-:

' . الإجراء الخاص بجلب كل أسماء المستخدمين ووضعهم في قائعة المستخدمين . Public Sub UsersIDs()

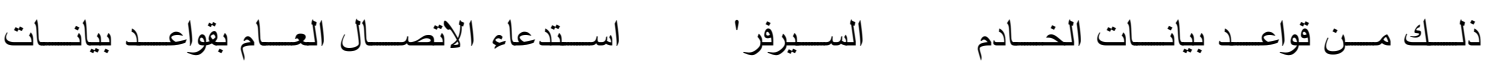

Try

Me.Cursor = Cursors. WaitCursor

'معرفة حالة الاتصال العام فإذا كان غير مفتوح

If GSQLConnection.State <> ConnectionState.Open Then

'عندها استدعي إجراء الاتصال العام من مديول الاتصال

GeneralConnection()

End If

If GSQLConnection.State = ConnectionState.Open Then

GDR = Nothing 
GSQLCmd.CommandType $=$ CommandType. Text

GSQLCmd.CommandText = "SELECT ${ }^{*}$ FROM Users"

GDR = GSQLCmd. ExecuteReader

Do While GDR.Read()

CmbUserName.Items.Add(GDR.GetSqIString(0))

Application.DoEvents()

Loop

GDR.Close()

GSQLConnection.Close()

Me.Cursor $=$ Cursors. Default

End If

Catch

Finally

Me.Cursor $=$ Cursors. Default

GSQLConnection.Close()

End Try

End Sub

[/code]

النافذة كما يلي والآن نستدعي الإجراء الأخير في حدث تحميل

Call UsersIDs() 


\section{المصادر}

إد واهلن ، 1998 ـ علم نفسك 8 ORACLE في 21 يومـا، الدار العربيـة للعلوم، مركز التعريبب

م/وائل إبراهيم الغنيمي ، 2000 ـ أساسيات شبكات الحاسب ، دار الراتب الجامعية، بيروت-لبنان.

دايتر كارول ، 2000 ـ أوراكل 8 بايبل ، دار الفاروق للنشر والتوزيـع / مركز التعريب والترجمـة ،

كوليتزيك بيتر ; بول دروسي ، 2001 ـ أوراكل ديفلوبر (ORACLE Developer) النماذج والتقارير

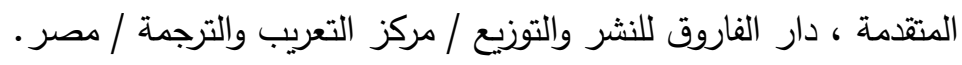

كوليتزيك بيتر ; بول دروسي ، 2001 ـ أوراكل ديزاينر (ORACLE Designer) ، دار الفاروق للنشر

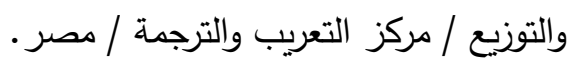

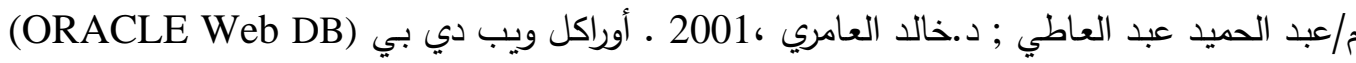

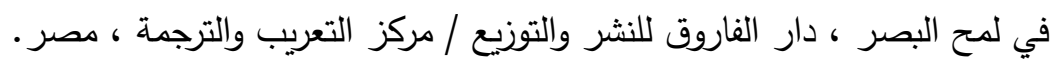

[7]. Aptech Certified (Computer Professional), 2001. ORDBMS Applications for the Web Oracle 8I.

[8]. Aptech Certified (Computer Professional) ,2001. Client Server Computing with Oracle 8I \&Developer 6.0. 
الملحق 1

التقارير الناتجة عن العمليات الاحصائية التي اجريت في النظام

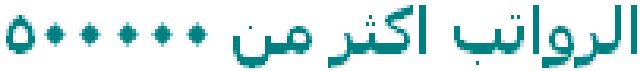

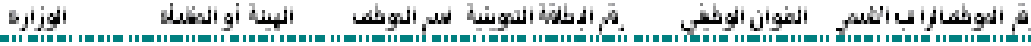

\begin{tabular}{|c|c|c|c|c|c|}
\hline ألـنائاة واللنفن & رية الـلنة الثنت اللى & خلا سلبلن & ino & هـt. & $797 \ldots$ \\
\hline الــناعة والهنلفن & رية النفال الثنت اللى & غnj ند & $1: 0$ & مينسف & $y_{1} \ldots$ \\
\hline 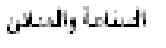 & شركة اللـنت البنوبة & 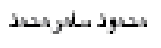 & $177 \mathrm{~V}$ & كتب & $10 \ldots$ \\
\hline الـناعدة والهنلان & شركة اللـنت البنوبة & שر tar & in & ل الثرس & $A \cdot B \cdots$ \\
\hline ألـنادة والهنلفن & شركة اللسنت ألبنوبدة & لرن زأس & וn & 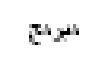 & ri.... \\
\hline ألـنادة والهنلان & شركا اللستت ألبنوبة & عنلن سلين & Invy & ندير & $170 \ldots$ \\
\hline ألـناعدة والهنلان & شركة اللـانت البنوبة & 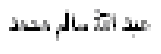 & $10 \mathrm{y}$ & كت & $10 \ldots$ \\
\hline 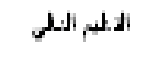 & حلفية الكائل & 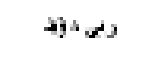 & $0 \times 770$ & 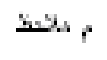 & $y_{1} \ldots$ \\
\hline الـ لئب, ألـلم & حلنية الك وهل & عر أشد فلتر & a & لهنفس & $0 \ldots$ \\
\hline
\end{tabular}

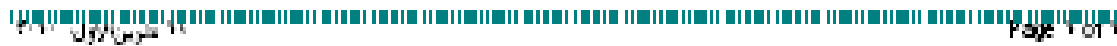




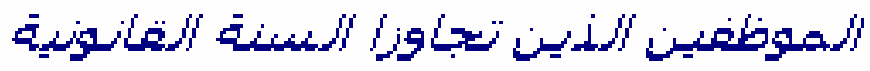

\begin{tabular}{|c|c|c|c|c|}
\hline -Linalig/ empll & 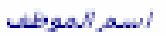 & إسي الولادة & Nubeg $A$ vewis $A$ & 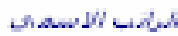 \\
\hline 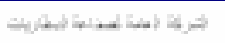 & 20 & mandere & $2 x$ & $T+\ldots$ \\
\hline 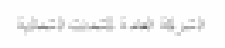 & $m$ & restentes & 20 & *a... \\
\hline 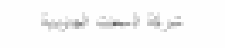 & 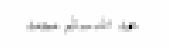 & motaces & +3 & $10 \mathrm{m.}$ \\
\hline 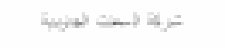 & 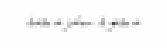 & inchest & +3 & $10 \ldots .$. \\
\hline
\end{tabular}




\section{الموظفين حسب تاريخ الميلاد}

\begin{tabular}{|c|c|c|c|c|c|}
\hline أنوزارك & انيدة أو الينافل & 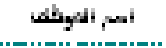 & مريخ انولى 6 & 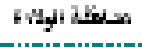 & انزاب ألامهن \\
\hline أحساعل والاشعن & 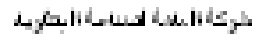 & 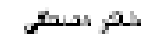 & 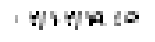 & نو & 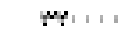 \\
\hline أحسعا، والاشعن & 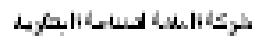 & عل مطاط & WVIAT: & نو & $42 \ldots$ \\
\hline 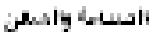 & 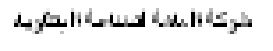 & أشدة لبك & $\forall V / V / A>2$ & نوA & sx. \\
\hline أحسعاك والاشعن & 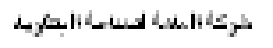 & 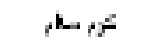 & $\mid$ MIY)/A:L & نو & 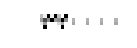 \\
\hline 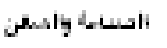 & 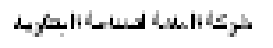 & أشد نرا & VV V/A & نو & sx. \\
\hline أحساعا، والامن & 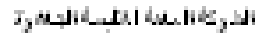 & d. & $: \theta / Y / A M A$ & - +وA & \& $\cdots \cdots$ \\
\hline أحساعا، والمبعن & 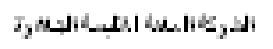 & 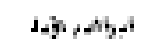 & PIYAR & نو & s.... \\
\hline أحساعا والاشعن & 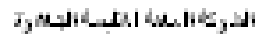 & 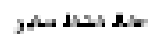 & 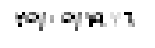 & - & " \\
\hline 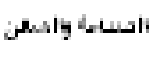 & 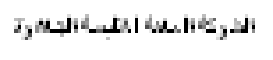 & קع & 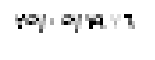 & +4 & , ' \\
\hline 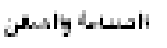 & 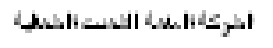 & 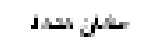 & $M / E / A \times 1$ & نوA & r..... \\
\hline 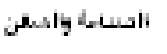 & 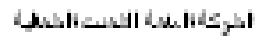 & حسن המי & $a / \cdot Y / A$ & نو & 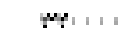 \\
\hline أاستعا، والاشن & 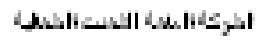 & خمادلدير & $P / 1 / 9 / 4: 1$ & نوء & ห⿻コ一... \\
\hline أاتساعي والهمن & خركانلسانتاشوبال & بر بر & $1 t / V / A t \gamma$ & sit & 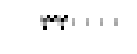 \\
\hline أحساعا والاشعن & 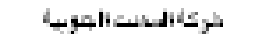 & 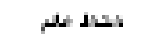 & $M / Y / A \times 7$ & بن & met... \\
\hline أنساعا، والدمن & شيكانلستالموبل، & 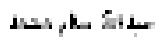 & 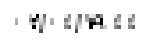 & ati & s..... \\
\hline 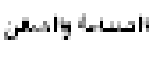 & خركانلسنافيوبة & نيا ني & YO/. 1/A & بن & 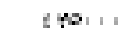 \\
\hline أستعا، والمعن & خركانلسنتاموبل & بن فئس & 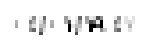 & بن & $19 \ldots$ \\
\hline أحساعا، والاشعن & 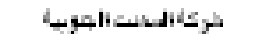 & علر بلم & $M / Y / A \times 7$ & بن & “ \\
\hline ناحسعا، والدمن & خركانلسنتألهوبة & يدول سمرنشد & 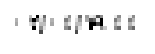 & ب د & s...... \\
\hline 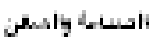 & شيكانلست المهوبة & علدز aلدj & YHIVIAOS & Al & no.... \\
\hline 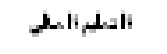 & حلسدانالمودل & 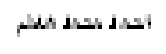 & YI/ E/AXA & ندو & (بה \\
\hline 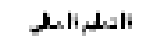 & حسنة|الانودل & ב. & YH/. ब/AX & 4y & y..... \\
\hline 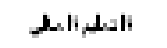 & حمبدانامودل & تار أشد لقد & $1 / 4$ บy & ن-y & Yo.... \\
\hline
\end{tabular}

II 
وزارة الصناعة والمعادن

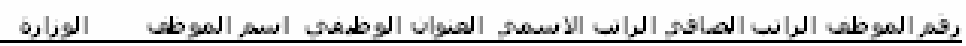

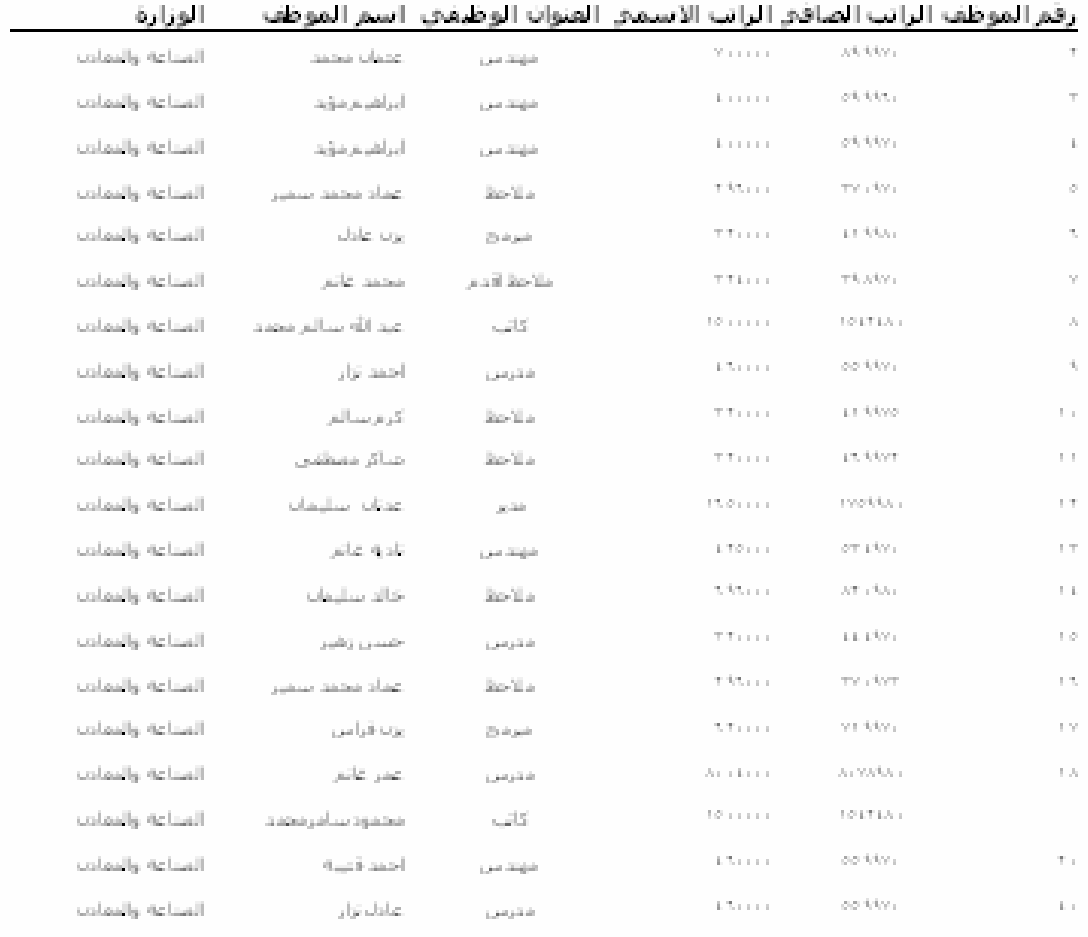

